\title{
Réflexion sur les violences extrêmes : Purifier et détruire, de Jacques Sémelin
}

\section{Elwis Potier}

\section{(2) OpenEdition \\ 1 Journals}

\section{Édition électronique}

URL : http://journals.openedition.org/conflits/2044

DOI : 10.4000/conflits.2044

ISSN : $1777-5345$

Éditeur :

CCLS - Centre d'études sur les conflits lilberté et sécurité, L'Harmattan

\section{Édition imprimée}

Date de publication : 1 mars 2006

Pagination : 165-172

ISBN : 2-296-00622-1

ISSN : 1157-996X

\section{Référence électronique}

Elwis Potier, «Réflexion sur les violences extrêmes : Purifier et détruire, de Jacques Sémelin », Cultures \& Conflits [En ligne], 61 | printemps 2006, mis en ligne le 17 mai 2006, consulté le 30 mars 2021. URL: http://journals.openedition.org/conflits/2044 ; DOI : https://doi.org/10.4000/conflits.2044

Ce document a été généré automatiquement le 30 mars 2021.

Creative Commons License 


\title{
Réflexion sur les violences extrêmes : Purifier et détruire, de Jacques Sémelin
}

\author{
Elwis Potier
}

1 Sémelin, J., Purifier et détruire. Usages politiques des massacres et génocides, coll. «La Couleur des idées », Paris, Le Seuil, 2005.

2 Jacques Sémelin poursuit sa vaste exploration de la violence en menant une réflexion approfondie sur ses formes les plus extrêmes, les plus négatrices : la violence dans ce qu'elle a de plus macabre, de plus terrifiant, de plus absurde ou de proprement sidérant. L'auteur de ce grand livre oriente sa recherche vers ce qui parait toujours plus inintelligible, l'énigme éminemment politique ainsi nommée « destructivité humaine de masse ", pour comprendre les mécanismes à l'œuvre, les conditions et les processus qui aboutissent aux meurtres de masse.

3 Après avoir centré ses recherches sur la non-violence, initiant des travaux remarquables conduits au sein de l'Institut de recherche sur la résolution non violente des conflits ${ }^{1}$ et précisément sur la résistance civile en Europe sous le nazisme ${ }^{2}$, études qui ont contribué de manière décisive à porter un autre regard sur la Résistance ${ }^{3}$, Jacques Sémelin, en historien et penseur de la Résistance civile, montre dans cet ouvrage comment se mettent en place les processus de purification et de destruction du « corps social » afin d'analyser les « usages politiques des massacres et des génocides». Tel est le projet annoncé par le sous-titre, ce qui revient en l'occurrence à traquer, notamment dans les " passages à l'acte », ce qui précisément résiste à l'analyse.

4 Si le titre de l'ouvrage rappelle inévitablement Surveiller et Punir de Michel Foucault, ce n'est pas seulement en raison de son homophonie. Il indique de la sorte une des références dont l'enquête ici engagée, parce qu'elle vise à saisir les liens entre violence et pouvoir, est d'une certaine façon une continuation, un prolongement possible, même si elle ne reprend pas, ou si peu, les analyses de Foucault. L'auteur s'en explique d'ailleurs dès son introduction intitulée "Comprendre ${ }^{4}$ ?": "A l'évidence, le pouvoir politique, qui fait la matière de ce livre, n'apparaît pas de même nature que ceux des XVII' et 


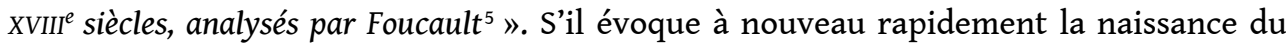
«bio-pouvoir » dans le dernier chapitre du livre, non sans avoir très justement relevé auparavant l'expression de "populicide» employée par Babeuf lors de la Révolution française, ces questions de "population», de "biopolitique» et de génocide, retravaillées ensuite par Foucault dans ses cours au Collège de France, ne font pas ici l'objet d'autres commentaires. Ce sont sans doute le titre évocateur et le caractère très explicite de la référence à Foucault qui peuvent parfois susciter de telles attentes.

Les deux verbes, "purifier » et " détruire ", désignent exactement les deux facettes du même pouvoir dont l'auteur entreprend d'analyser les manifestations à travers la comparaison des grands massacres historiques du $\mathrm{xx}^{\mathrm{e}}$ siècle, principalement la Shoah, les nettoyages ethniques de l'ex-Yougoslavie et le génocide des Tutsi au Rwanda, en élargissant, selon les problématiques traitées, à d'autres exemples comme les génocides arménien et cambodgien. Il ne s'agit donc pas d'une archéologie du génocide, ni même d'un essai au sens philosophique du terme. Cette étude, qui prend la forme d'une synthèse de plusieurs années de recherches au CNRS, ne cherche pas à faire une relecture philosophique de l'histoire, c'est d'ailleurs ce qui en fait sa qualité première. L'auteur reste clairement dans une démarche d'enquête empirique et donne au lecteur tous les éléments de méthode utiles lui permettant de vérifier ou de juger sur pièces (on se reportera profitablement à l'introduction et aux annexes).

6 Il s'agit bien plus d'analyser, dans une approche transdisciplinaire, les différentes formes historiques de massacres collectifs suivant une démarche comparative rigoureuse en s'appuyant sur les nombreux travaux d'historiens et confrontant les différentes lectures et interprétations. Cela tend à situer Jacques Sémelin, comme il s'en réclame lui-même, davantage dans la lignée d'historiens comme Léon Poliakov et Yves Ternon. La qualité du travail historiographique n'a d'égal que la richesse de la documentation sur laquelle se fonde le questionnement. L'ouvrage, à cet égard, fait figure d'outil de travail indispensable, qui plus est, passionnant. L'autre intérêt indéniable tient à la portée des analyses et à la compréhension qu'il donne de la complexité des phénomènes décrits, une compréhension qui doit beaucoup à la diversité des références théoriques et à la posture singulière du chercheur.

7 Jacques Sémelin a été psychosociologue avant d'être professeur à Sciences Po. Il en a gardé un attrait pour les approches pluridisciplinaires, ce qui l'amène à croiser les regards psychologique, psychosociologique, anthropologique, philosophique, tout en maintenant le cap d'une sociologie politique, que l'on peut, sans hésiter qualifier de compréhensive. Cela revient, outre la filiation à Weber, à faire appel à des éclairages différents "sans réduire les phénomènes sociaux à des processus psychologiques ${ }^{6} »$. On retrouve ainsi avec plaisir des auteurs qui ont nourri sa réflexion et jalonné ses écrits depuis son premier livre publié en 1983 Pour sortir de la violence ${ }^{7}$ : Arendt, Freud, Bettelheim, Fornari, Milgram, etc. Dans le cinquième chapitre par exemple ( Les vertiges de l'impunité ${ }^{8} »$ ), les pages consacrées au « crime d'obéissance » reprennent les expériences de Milgram et Zimbardo avant de revenir plus longuement sur « la banalité du mal » et de revisiter les thèses d'Arendt. Cette ouverture pluridisciplinaire stimule la réflexion critique et permet de rendre compte des convergences et des divergences entre les différentes approches, d'autant que l'auteur, méfiant vis-à-vis du jargon et de l'enflure verbale, se garde bien de glisser vers une "esthétique de l'horreur ${ }^{9}$ » et parvient à exprimer les différents points de vue, y compris le sien, dans un style simple et précis. 
8 Les noms de psychologues, de philosophes et autres écrivains viennent naturellement compléter l'impressionnante liste des historiens, sociologues ou politistes de divers horizons qui alimentent la réflexion tout au long de l'ouvrage. L'étendue de l'investigation, par cette pluralité des approches et des thématiques, peut poser la question des limites de son champ, autrement dit, de la dispersion, mais il n'en est rien: la complexité de "l'abîme génocidaire" nécessite une telle démarche. C'est davantage l'objet même de cette recherche qui pose inévitablement problème, à commencer par les termes choisis et les notions qui le désignent. Jacques Sémelin en est pleinement conscient et décide de s'en tenir au terme de "massacre ", terme qu'il qualifie paradoxalement de «minimal » et qu'il propose de définir en tant que « forme d'action le plus souvent collective de destruction de non-combattants ${ }^{10} »$. La question du génocide est renvoyée en fin d'ouvrage et fait l'objet d'une analyse critique de ses usages et instrumentalisations, depuis son invention par Raphael Lemkin en 1944 et la définition du crime de génocide par la Convention de l'ONU en 1948, qui a marqué les premières études en sciences sociales sur le sujet. Cette clarification ne pourra se faire sans revenir sur la notion de massacre collectif, et sans reprendre les débats sur l'emploi du terme « Holocaust » et de l'expression «nettoyage ethnique ». Le parti pris de Sémelin veut donc que l'analyse de ces «usages politiques" ne puisse se faire qu'après avoir enquêté sur les processus qui aboutissent aux massacres. Cette mise à distance à laquelle correspond le report du travail sur la définition de l'objet d'étude répond bien de la démarche empruntée par le chercheur soucieux d'adopter une juste posture face à cet objet difficile, s'il en est. Ce qu'il nomme « l'abîme génocidaire » est en effet un objet massif (c'est cet aspect qui est le premier discriminant), sidérant, " hideux ", qui oblige à une distance critique y compris envers les mots eux-mêmes, mots par lesquels le fantasme se mêle à la réalité.

La construction de l'ouvrage est donc remarquable à plus d'un titre. Chaque chapitre s'ouvre sur un éclairage particulier, un nouvel angle d'analyse, qui prolonge le précédent et renouvelle le questionnement. L'auteur nous convie ainsi à plusieurs temps d'analyse qui sont autant de niveaux d'interprétation, dans l'articulation desquels progresse une réelle compréhension du phénomène génocidaire. Il serait donc vain, on l'aura compris, de vouloir résumer une telle somme, tout au plus pouvons-nous donner quelques indications afin de rendre une idée de l'ensemble et nous interroger sur quelques points.

10 Le fil rouge qui dessine le parcours emprunté par l'auteur pour affronter la question du pouvoir de destruction est le passage à l'acte, «mouvement de bascule du fantasme à l'action ${ }^{11} »$. Ce «moment » décisif semble insaisissable puisqu'il n'y a pas « un » acte ou " une » décision comme il ne peut y avoir « une seule » cause. Il sera alors appréhendé comme "processus de bascule, complexe, imbriquant des dynamiques collectives et individuelles, de nature politique, sociale, psychologique, etc. ${ }^{12} »$.

11 Le premier chapitre ${ }^{13}$ s'ouvre naturellement sur une revue critique des théories qui privilégient un seul facteur, qu'il soit économique, culturel, démographique ou psychologique. Cette mise au point est d'emblée nécessaire tant les explications hâtives sont légion, comme l'interprétation très courue qui voudrait que la violence politique et les massacres collectifs traduisent une dilution ${ }^{14}$ du politique, celle des institutions et des Etats-nations. Sémelin rappelle à juste titre qu'« écrire l'histoire, c'est ouvrir le champ des possibles, en se méfiant de toute interprétation causale simpliste et déterministe du passé $^{15} »$. L'enchevêtrement des causes est certainement plus pertinent mais 
l'accumulation des « causes » possibles ne peut faire l'économie des significations de la situation pour les acteurs impliqués. «Pour vivre, les hommes ont besoin de donner du sens à leur existence. Pour tuer, il en est de même. Ce tremplin mental vers le meurtre de masse repose sur les interactions constantes entre imaginaire et réel, à travers lesquelles toute limite est abolie $^{16} »$.

Ces considérations sur les significations sociales amènent l'auteur à souligner la puissance de l'imaginaire, un imaginaire finement analysé à travers les thèmes de l'identité, de la pureté et de la sécurité, dans ses rapports à l'idéologie qui le relie au réel. Cette dynamique se traduit par le passage de l'angoisse collective à la peur intense à l'égard d'un ennemi, peur qui va pouvoir faire l'objet de manipulations. Après avoir décrit l'imaginaire de toute puissance et de destruction et la logique identitaire justement résumée par la formule "détruire le "eux" pour sauver le "nous"17》, Sémelin s'attarde, dans le deuxième chapitre ${ }^{18}$, sur l'intention à partir de laquelle se construit le « discours incendiaire » et « la violence sacrificielle ». L'analyse porte sur la nature du discours, les conditions de son élaboration et de sa légitimation, notamment par la convergence des registres intellectuel, politique, religieux et social. Le chapitre suivant sur le « contexte international, guerre et médias ${ }^{19}$ » se tourne vers les facteurs externes aux pays pour comprendre comment s'effectue la "transformation du processus de violence en acte de guerre ». Le quatrième chapitre sur « les dynamiques du meurtre de masse ${ }^{20}$ » est une description des "pratiques collectives» de massacre et des différents modes d'adhésion, sinon de participation, de la société. Le rôle déterminant des acteurs étatiques et para-étatiques est alors replacé dans sa juste mesure.

Insistons au passage sur un point crucial: il est nécessaire, et même salutaire, de rappeler, comme le fait Sémelin, qu'il n'y a pas de violence «spontanée » des masses ${ }^{21}$ comme il n'y a pas de "passivité » absolue des populations ${ }^{22}$. La fin de cette partie dresse un tableau à la fois édifiant et contrasté des "morphologies de la violence extrême » et des types de destruction qui s'achève par la question fondamentale et critique à nos yeux de «l'autonomisation ${ }^{23}$ » du meurtre de masse. Voilà quelques unes des nombreuses idées qui ne manqueront pas d'éveiller la conscience. De même que nous nous abstenons de rentrer dans les faits historiques qui n'ont d'intérêt que remis en perspective, ce survol très rapide du «cœur» de l'ouvrage n'a pour but que de renvoyer au « corps » du texte en espérant donner envie de le lire.

La partie sur "les vertiges de l'impunité » est l'occasion pour Sémelin de centrer l'analyse sur le «moment ", le «noyau dur » du passage à l'acte, et d'aller, en quelque sorte, au bout de son raisonnement. Au fil des pages de cet avant-dernier chapitre, en suivant les interrogations et les considérations sur la rationalité et le délire, puis sur la " jouissance de la cruauté », on arrive dans une «zone grise ${ }^{24}$ » qui semble aboutir à une impasse, à moins que ce ne soit le signe de notre libre arbitre.

Le dernier chapitre, synthèse sur les " usages politiques des massacres ", est à part. Il est en partie consacré, nous l'avons dit, à une réflexion sur la notion de génocide en lien avec d'autres notions proches. A ce propos, la perspective que donne l'auteur du «nettoyage ethnique» est détonante et nous paraît viser juste: le "nettoyage ethnique ", apparaît selon lui, comme "une nouvelle forme d'ingénierie sociale qui consiste [...] à découper le peuple rebelle ${ }^{25}$ ». Mais on peut, en revanche, s'interroger sur la place qu'il donne par la suite au terrorisme et sur l'intérêt d'engager en toute fin de volume une réflexion sur cette question. Les cas historiques évoqués - et le 11 septembre 2001 y prend, bien évidemment, toute sa place - n'ont que peu de rapport avec les cas de 
génocides précédemment cités. Bien sûr, on peut considérer que les actes terroristes, ou désignés comme tels, provoquent des massacres mais peut-on, pour autant, rester sur une définition «minimale» pour justifier toute comparaison? L'auteur, bien entendu, discute de l'usage du terme et cite les chercheurs qui font autorité en la matière ${ }^{26}$, mais il ne suit manifestement pas les avertissements de Didier Bigo pour qui "ce n'est pas un concept utilisable par les sciences sociales et la stratégie $e^{27}$ ", et préfère s'en remettre aux conceptions d'Isabelle Sommier (la " violence totale ${ }^{28}$ )), chez qui il trouve la justification de ce rapprochement avec le génocide. Il faut dire que ce dernier chapitre est également en grande partie consacré à la typologie que Sémelin a conçue au terme de sa recherche. Il distingue trois types de logiques politiques des massacres que sont la soumission, l'éradication et l'insurrection. Il se trouve que le terrorisme constitue la principale illustration de la logique insurrectionnelle. Peut-on expliquer avec les mêmes concepts la violence génocidaire et la violence terroriste? Des massacres peuvent, certes, participer d'une logique d'insurrection, mais faut-il toujours parler de «massacres de masse » ? Peut-être ne s'agit-il plus de la même « masse », non plus des mêmes « massacres » ni de la même « destruction ".

La conclusion ouvre des pistes intéressantes pour apporter des réponses concrètes aux problèmes soulevés. Soucieux de mener des recherches utiles, et fidèle à une éthique de la responsabilité, Jacques Sémelin propose des axes de travail prometteurs tant sur le plan des sciences sociales que sur celui de l'action internationale.

\section{NOTES}

1.. Publiés dans la revue Alternatives non violentes.

2.. Son ouvrage Sans armes face à Hitler (préfacé par Jean-Pierre Azéma, Paris, Editions Payot, 1989), né d'une interrogation « de nature éthique et stratégique », a fait date sur la question.

3.. Ces différentes études ont largement contribué à ouvrir le champ des sciences politiques par l'élaboration de nouvelles notions telles la « dissuasion civile » et la constitution de ce qu'il conviendrait d'appeler un « répertoire » des actions non violentes.

4.. Sémelin J., Purifier et détruire, op. cit., pp. 15-23.

5.. Ibid., p. 21.

6.. p. 286.

7.. Sémelin J., Pour sortir de la violence, Paris, Les Editions ouvrières, 1983.

8.. Sémelin J., Purifier et détruire, op. cit., pp. 285-364.

9.. Ibid., p. 22.

10.. Ibid., p. 19.

11.. Ibid., p. 39

12.. Ibid., p. 16

13.. Ibid., pp. 25-74 
14.. Ne confondons pas « dilution » et " délitement », Sémelin est convaincu qu' " aucune société n'est à l'abri de tels processus dès lors qu'elle commence à se déliter » (Ibid., p. 25).

15.. Ibid., p. 87.

16.. Ibid., p. 287.

17.. Ibid., p. 70.

18.. Ibid., pp. 75-133.

19.. Ibid., pp. 135-200.

20.. Ibid., pp. 201-284.

21.. Ibid., p. 203.

22.. Ibid., p. 267.

23.. Ibid., p. 284.

24.. Expression de Primo Levi, dans Les Naufragés et les Rescapés. Quarante ans après Auschwitz, Paris, Gallimard, 1989.

25.. p. 403.

26.. pp.416-419.

27.. Bigo D., « L'impossible cartographie du terrorisme », Cultures \& Conflits, automne 2001.

28.. Voir Sommier I., Le Terrorisme, Paris, Flammarion, coll. « Dominos », 2000.

INDEX

Mots-clés : génocide, massacre de masse

\section{AUTEUR}

\section{ELWIS POTIER}

Elwis Potier est psychosociologue, auteur de l'article « L'imaginaire du contrôle des foules dans l'armée de terre française », Cultures \& Conflits n56, hiver 2004. 